\title{
Photodynamics of an excited triplet state in the presence of molecular oxygen cluster
}

\author{
Yoshinobu Nishimura*
}

Graduate School of Pure and Applied Sciences, University of Tsukuba, 1-1-1 Tennoudai, Tsukuba, Ibaraki 305-8571, Japan

\begin{abstract}
Bulk nanobubble is one of the nanoscopic gaseous state found in the solution. We measured transient absorption spectra of eosin $\mathrm{Y}$ in the excited triplet state $\left({ }^{3} \mathrm{EY}^{2-*}\right)$ to elucidate differences in the dissipation process of the bulk nanobubble of oxygen molecule at air pressure. The time dependence of the oxygen dissipation process was classified three time domains (P1, P2 and P3). The comparison of ordinary bubbling method gave different results at $\mathrm{P} 3$ in contrast to similar results at $\mathrm{P} 1$ and P2, indicating the existence of characteristic dissolving state in the case of nanobubble.
\end{abstract}

Recently much attention has been focused on gas nanobubbles in the aqueous solution because of various applications such as nanoscopic cleaning in semiconductor process, promotion of vegetable growing, wastewater treatment and froth flotation. ${ }^{1-6}$ Gas nanobubbles consist of two kinds of gas state referred to as bulk and surface nanobubbles locating in a bulk solution and on the solid surface, respectively. The former can be measured by dynamic light scattering (DLS) methods although the latter is investigated by AFM, FTIR, QCM and SPR. There are few methods to study bulk nanobubbles. Since an excited state is allowed to undergo oxygen quenching photochemically, we selected the transient absorption technique to elucidate quenching activity of dissolved oxygen gas, i.e. bulk nanobubble of oxygen molecule, which appears in the lifetime of an excited state. If

*To whom correspondence should be addressed.

E-mail: nishimura@chem.tsukuba.ac.jp the quenching rate constant is known, oxygen concentration dissolved in solution can be estimated according to Stern-Volmer equation. In the present study, the decrement of formed oxygen nanobubbles by a nanobubble generator without a seal of a sample cell was observed by measuring $\mathrm{T}_{0}-\mathrm{T}_{1}$ absorption decay of eosin $\mathrm{Y}$. The present report includes two different methods of oxygen dissolution into a solution; nanobubble generation and conventional bubbling.

The time development of oxygen concentration included in the solution just after stopping nanobubble generation showed three characteristic periods referred to as P1, P2 and P3 as a function of elapsed time (Fig. 1). These phenomena at each period can be associated with the situation of oxygen nanobubbles since the time dependence of oxygen concentration was estimated from the quenching of ${ }^{3}\left(\mathrm{EY}^{2-}\right)^{*}$ by oxygen dissolved in solution. P1, which was observed in the first period of time, showed very rapid decay having time constant of about half an hour independent of oxygen injection method. The oxygen concentration of $1.6 \times 10^{-3} \mathrm{M}$ in both methods just after stopping oxygen injection gave oversaturated concentration of oxygen since it is $0.28 \times 10^{-3} \mathrm{M}$ under atmospheric pressure.

The P2 period following P1 showed irregular fluctuation in oxygen concentration rather than the phenomena observed in $\mathrm{P} 1$, which showed rapid decay of oxygen concentration. These differences were observed in both methods up to $10 \mathrm{~h}$ elapsed. The oxygen concentration was varied from $0.9 \times 10^{-3} \mathrm{M}$ to $1.3 \times 10^{-3} \mathrm{M}$, which are still oversaturated condition of oxygen molecules dissolved in the solution. If molecular oxygen dissolved is only released to the air, 
such fluctuation of the concentration of oxygen cannot occur under atmospheric pressure. There seems to be something different quenching mechanism of ${ }^{3}\left(\mathrm{EY}^{2-}\right)^{*}$ by oxygen in cluded in the solution.

The final period P3 gave a single exponential decay having lifetimes of $13 \mathrm{~h}$ and $4.9 \mathrm{~h}$ for nanobubble and needle methods, respectively. Interestingly, the start time of P3 was independent of injection methods to be $10 \mathrm{~h}$ elapsed. The oxygen concentration in both methods achieved to about $0.3 \times 10^{-3} \mathrm{M}$, which is equilibrium condition under atmospheric condition. This means that oversaturated situation was broken up through release of oxygen molecules dissolved in the solution to the atmosphere thermodynamically. The origin of differences in time constant between two methods may be related to the thermodynamic stability of nanobubbles in the solution.

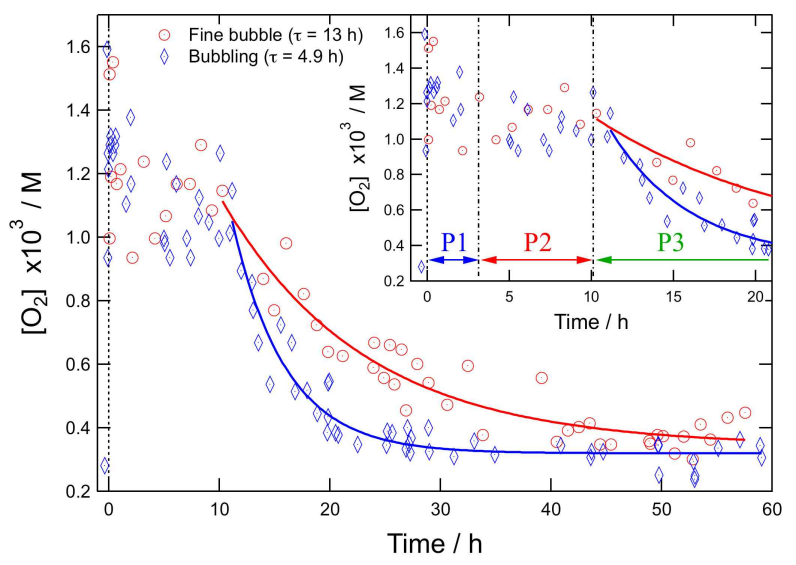

Figure 1. $\mathrm{KCl}$ effect on changes in $\left[\mathrm{O}_{2}\right]$ as a function of elapsed time.

To determine the size of nanobubbles is the key to investigate properties and behaviors of nanobubbles in the solution, whereas the quenching of ${ }^{3}\left(\mathrm{EY}^{2-}\right)^{*}$ by oxygen molecules dissolved in the solution allows us to determine the concentration of oxygen leading to the luck of information of nanobubbles generated. Obtained size distribution of nanobubbles by using DLS at $0.4 \mathrm{~h}$ after stopping nanobubble generator gave three major peaks at $150 \mathrm{~nm}, 500 \mathrm{~nm}$ and 10 $\mu \mathrm{m}$ as shown in Fig. 2. The distribution peak located at $500 \mathrm{~nm}$ showed the most intense one having about $200 \mathrm{~nm}$ of the full width at the half maximum (fwhm). The fwhm of $150 \mathrm{~nm}$-species was relatively narrower than that of $500 \mathrm{~nm}$, indicating that it may be a minor component observed. This assumption is also applicable to the observation of $10-\mu \mathrm{m}$ component. The distribution shape of three components were constant up to $1.4 \mathrm{~h}$, which corresponds to P1. Interestingly, $150-\mathrm{nm}$ species was not observed constantly. This may come from the extremely narrower fwhm than other species. After that period, the peak size slowly increased up to $6.2 \mathrm{~h}$, which corresponds to P2. The fwhm in size distribution was changed irregularly like peak size. Such irregularity of peak size and size distribution may indicate the instability of nanobubbles in the solution, which is consistent with the irregularity of oxygen concentration determined by quenching experiment at P2 time region. After $21.4 \mathrm{~h}$ corresponding to $\mathrm{P} 3$, the remarked changes in the fwhm of size distribution was found to be more than $1 \mu \mathrm{m}$. The $500-\mathrm{nm}$ species gradually increased to the peak size of $1 \mu \mathrm{m}$. It is noteworthy that about 100 -nm species was more often observed relative to P1 and P2. Furthermore, more than $10-\mu \mathrm{m}$ species increased its intensity relative to P1 and P2. These results could be explained by the collapse of nanobubbles or some artifacts due to the limit of the apparatus.

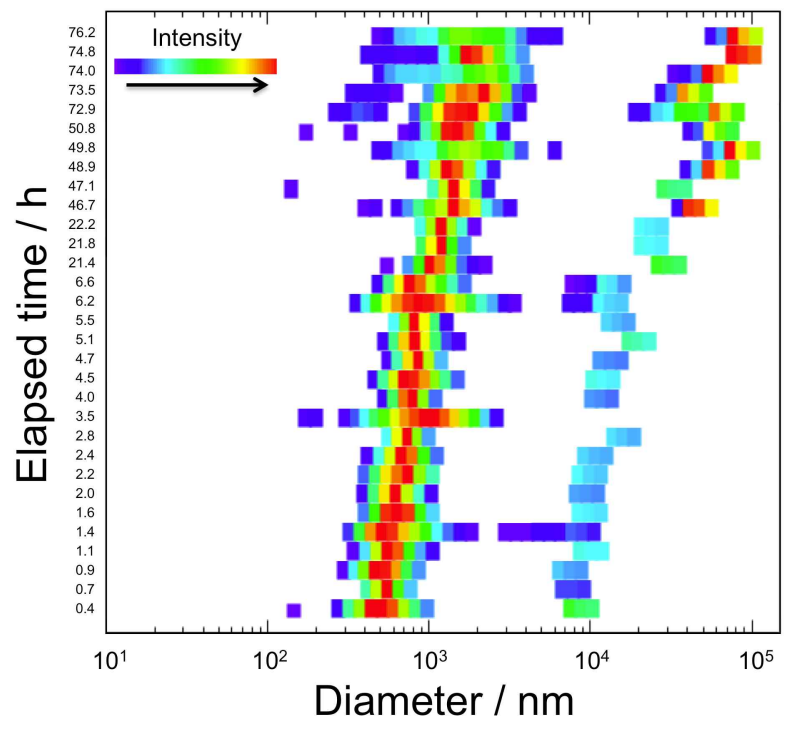

Figure 2. Size distribution obtained by DLS as a function of elapsed time.

Laplace pressure predicts that the inner pressure of nanobubbles increases with decreasing the diameter, 
which means subsequent disappearance of nanobubbles. ${ }^{7}$ However, we are interested in the shape of nano cluster produced by a nanobubble generator. If the shape is far from the glove, the inner pressure may depend on each shape of nanobubbles. We assume that a nanobubble consists of only molecular oxygen although ordinary bubbles have a free space, which allows us to postulate the existence of gas phase in it. Inner oxygen molecules may interact with each other by Wan der Waals force, whereas outer oxygen molecules interact with $\mathrm{H}_{2} \mathrm{O}$ through hydrogen bonding interaction. If so, each oxygen molecule may not sense any inner pressures. This mechanism enables us to release free oxygen molecule to bulk solution followed by quenching of ${ }^{3}\left(\mathrm{EY}^{2-}\right)^{*}$. The kinetics of oxygen releasing process may play an important role of time development of the lifetime of ${ }^{3}\left(\mathrm{EY}^{2-}\right)^{*}$.

Figure 3 shows the quenching mechanism of ${ }^{3}\left(\mathrm{EY}^{2-}\right)^{*}$ by oxygen. There are many kinds of shapes of oxygen molecules dissolved in water in the $\mathrm{P} 1$ region due to oversaturated oxygen molecules indicating that P1 is very unstable region. P2 also has various shapes of oxygen molecules including nano- or microbubbles as well as detached oxygen molecules solvated by $\mathrm{H}_{2} \mathrm{O}$, which are allowed to quench ${ }^{3}\left(\mathrm{EY}^{2-}\right)^{*}$. However, $\mathrm{P} 2$ shows fluctuation of $\left[\mathrm{O}_{2}\right]$ in contrast to $\mathrm{P} 1$. Nanobubbles having free space in the glove may collapse into smaller bubbles, which has no such a free space. Another nanobubbles may fuse other bubbles. Meanwhile, detached oxygen molecules may form into the bulk solution. These processes are considered to be non-equilibrium one, indicating that formation frequency of detached oxygen molecules is not constant. In the $\mathrm{P} 3$ region there may not be no oxygen clusters since time dependence of $\left[\mathrm{O}_{2}\right]$ showed the first order kinetics. Detached oxygen molecules are allowed to be released to the air having a constant rate. Differences in time constants in the $\mathrm{P} 3$ region between nanobubbles and bubbling may come from hydrated oxygen cluster in the $\mathrm{P} 1$ and $\mathrm{P} 2$ regions.

Consequently, transient absorption measurement of ${ }^{3}\left(\mathrm{EY}^{2-}\right)^{*}$ in the presence of oxygen molecules revealed three different dissolved state dependent on elapsed time in the open system where oxygen molecules are released to the air.

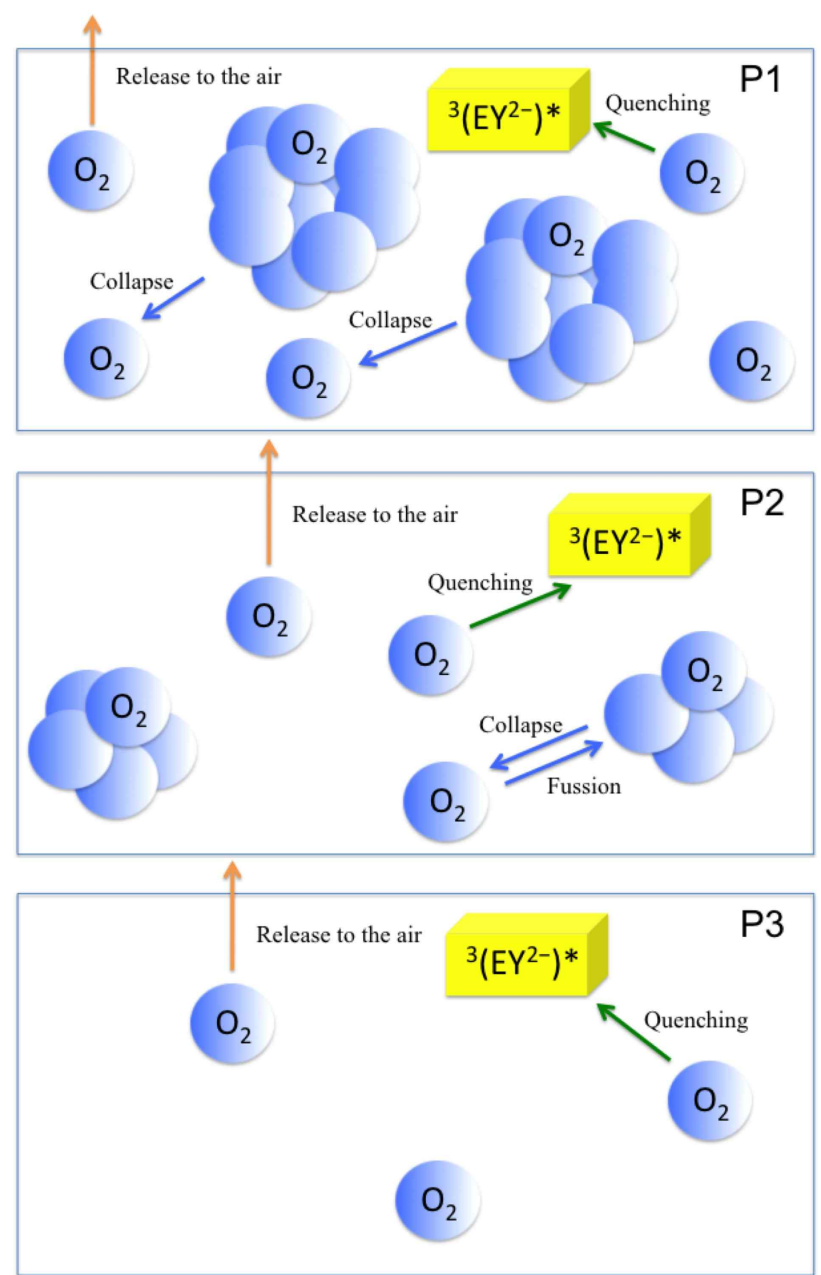

Figure 3. Plausible explanation of $\mathrm{P} 1, \mathrm{P} 2$ and $\mathrm{P} 3$.

KEYWORDS : Nanobubble, triplet quenching, oxygen cluster

Received November 27, 2013; Accepted December 3, 2013

\section{ACKNOWLEDGEMENT}

We are grateful to Prof. T. Arai for support of this research.

\section{SUPPORTING INFORMATION}

Experimental procedures; Transient absorption spectra and decay curves. This material is available free of charge via the Internet at http://photos.or.kr

\section{REFERENCES AND NOTES}

1. Seddon, J. R. T.; Lohse, D.; Ducker, W. A.; Craig, V. S. J. Chem. Phys. Chem. 2012, 13, 
2179-2187.

2. Ohgaki, K; Khanh, N. Q.; Joden, Y.; Tsuji, A.; Nakagawa, T. Chem. Eng. Sci. 2010, 65, 1296-1300.

3. Pan, G.; Yang, B. Chem. Phys. Chem. 2012, 13, 2205-2212.

4. Jin, F; Ye, X.; Wu, C. J. Phys. Chem. B 2007, 111, 13143-13146.
5. Creux, P.; Lachaise, J.; Graciaa, A.; Beattie, J. K. J. Phys. Chem. C 2007, 111, 3753-3755.

6. Takahashi, M.; Chiba, K.; Li, P. J. Phys. Chem. B 2007, 111, 11443-11446.

7. Ljunggren, S; Eriksson, J. C. Colloids Surf. A 1997, 129-130, 151-155. 